\title{
Tricotilomania: dificuldades diagnósticas e relato de dois casos
}

\author{
Trichotillomania: difficulties in diagnosis and report of two clinical cases
}

\begin{abstract}
Maria Cristina P. Lima', Érica Vasques Trench², Louise Lopes Rodrigues ${ }^{3}$, Lidiane Affonso de S. Dantas ${ }^{3}$, Gustavo Bigaton Lovadini, Albina Rodrigues Torres ${ }^{4}$
\end{abstract}

\section{RESUMO}

Objetivo: Relatar dois casos de tricotilomania, um transtorno psiquiátrico ainda subdiagnosticado e que pode estar associado a problemas sociais e clínicos relevantes. Pretende-se destacar as características clínicas, discutindo as implicações do diagnóstico precoce para a evolução dos pacientes.

Descrição do caso: Uma adolescente com diagnóstico de tricotilomania "pura" e outra menina cujo quadro estava associado ao transtorno obsessivo-compulsivo. Embora com o tratamento, a evolução de ambas tenha sido favorável, houve demora significativa para estabelecer o diagnóstico e encaminhá-las a um serviço de saúde mental, com prejuízos escolares e sociais.

Comentários: A tricotilomania difere dos quadros benignos e transitórios de arrancar cabelos observados nos primeiros anos de vida e ainda é subdiagnosticada. A vergonha dos sintomas observada nos portadores e o desconhecimento por parte dos profissionais de saúde contribuem para essa situação. O quadro pode ser grave, particularmente se acompanhado de tricofagia. Profissionais da saúde precisam identificar o transtorno precocemente e encaminhar as crianças para tratamento especializado antes das possíveis complicações clínicas e repercussões psicossociais.

Palavras-chave: tricotilomania; transtorno obsessivocompulsivo; criança.

\section{ABSTRACT}

Objective: To report two cases of trichotillomania, an underdiagnosed psychiatric disorder that may be associated with important social and clinical problems. The clinical features will be highlighted considering the implications of early diagnosis on patients' outcome.

Case description: An adolescent with isolated trichotillomania and another girl whose symptoms were associated with obsessive-compulsive disorder are described. Although both patients presented a favorable outcome with treatment, there was a significant delay in establishing the diagnosis and in referring them to a mental health service, leading to negative impact in educational and social domains.

Comments: Trichotillomania differs from the benign and transitory hair pulling habits observed in the first years of life, and it is still underdiagnosed. The embarrassment related the patients' symptoms and the lack of knowledge of health professionals contribute to this situation. The condition may be severe, particularly if associated with trichophagia. Health care professionals need to identify the disorder early and refer these children to specialized treatment before clinical complications and psychosocial problems occur.

Key-words: trichotillomania; obsessive-compulsive disorder; child.
Instituição: Departamento de Neurologia, Psicologia e Psiquiatria da Faculdade de Medicina de Botucatu da Universidade Estadual Paulista (Unesp), Botucatu, SP, Brasil

'Médica; Professora Doutora do Departamento de Neurologia, Psicologia e Psiquiatria da Faculdade de Medicina de Botucatu da Unesp, Botucatu, SP, Brasil

${ }^{2}$ Médica; Professora do Departamento de Neurologia, Psicologia e Psiquiatria da Faculdade de Medicina de Botucatu da Unesp, Botucatu, SP, Brasil

${ }^{3}$ Acadêmico de Medicina da Unesp, Botucatu, SP, Brasil

${ }^{4}$ Médica; Professora Doutora do Departamento de Neurologia, Psicologia e Psiquiatria da Faculdade de Medicina de Botucatu da Unesp, Botucatu, SP, Brasil
Endereço para correspondência:

Maria Cristina P. Lima

Rubião Júnior, s/n

CEP 18618-970 - Botucatu/SP

E-mail: mclima@fmb.unesp.br

Conflito de interesse: nada a declarar

Recebido em: 19/12/08

Aprovado em: 25/4/09 


\section{Introdução}

A tricotilomania foi descrita pela primeira vez em 1889 por Hallopeau ${ }^{(1)}$, sendo atualmente classificada entre os transtornos de hábito e de controle de impulsos ${ }^{(2)}$. Os pacientes referem sentir uma urgência ou necessidade incontrolável de arrancar os próprios pelos, principalmente os cabelos, podendo também envolver sobrancelhas, cílios, pelos pubianos ou de qualquer outra parte do corpo. Não raro, os pacientes ingerem os fios de cabelo arrancados ou parte desses, caracterizando a tricofagia ${ }^{(3)}$. Segundo o Diagnostic and Statistical Manual of Mental Disorders (DSM), $4^{\text {th }}$ edition ${ }^{(4)}$, a tricotilomania se caracteriza por comportamento recorrente de arrancar cabelos, com perda capilar perceptível; aumento da tensão imediatamente antes de arrancar o cabelo ou quando tenta resistir ao comportamento; prazer, satisfação ou alívio após arrancar os cabelos. O transtorno não é explicado por outro transtorno mental e não se deve a condições médicas gerais (por exemplo, outras condições dermatológicas) e causa sofrimento clinicamente significativo ou prejuízo no funcionamento social, ocupacional ou de outras áreas importantes na vida da pessoa.

Nos primeiros anos de vida, o comportamento de arrancar cabelos é usualmente uma manifestação clínica autolimitada, de pouca gravidade e evolução benigna, associada à busca de conforto e acompanhada de outros comportamentos com finalidade semelhante, como a sucção do polegar, por exemplo ${ }^{(5)}$. Sua ocorrência mais tardia, porém, pode estar relacionada a eventos estressantes e ser acompanhada de prejuízos importantes. Um estudo que avaliou 133 crianças e adolescentes entre dez e 17 anos encontrou elevada prevalência de sintomas depressivos e ansiosos ${ }^{(6)}$. Nessa população, o início mais tardio da tricotilomania foi um preditor para sintomas depressivos e ansiosos, independentemente da duração da doença ${ }^{(6)}$. Além de danos estéticos e psicossociais, podem ainda ocorrer complicações clínicas decorrentes da ingestão dos pelos e cabelos, uma característica comum nos portadores de tricotilomania ${ }^{(7)}$. O tricobezoar é uma massa formada a partir de pelos e cabelos deglutidos impactados no interior do trato gastrointestinal e pode assumir, muitas vezes, formas graves, nas quais o bolo de cabelo ingerido ocupa uma parte importante do intestino, causando dores abdominais, náuseas, vômitos, anemia, hematêmese, úlceras, pancreatite e até mesmo perfuração intestinal e abdome agudo obstrutivo ${ }^{(8)}$. Algumas vezes, esses tricobezoares se estendem da massa principal localizada no estômago até o cólon por meio de uma extensa cauda de fios, recebendo a denominação de Síndrome de Rapunzel, em alusão à princesa dos contos de fadas ${ }^{(9)}$.

A idade média de início do distúrbio varia entre nove e 13 anos, apesar de haver um grupo de início precoce, na fase pré-escolar ${ }^{(10)}$. Quando os sintomas aparecem em idades mais tardias, a tricotilomania é frequentemente associada a outras doenças psiquiátricas como depressão, transtorno obsessivocompulsivo e outros transtornos de controle de impulso, como o skin picking ${ }^{(10-13)}$. A prevalência de tricotilomania em crianças tem sido relatada em $1 \%$; entretanto, é possível que esse percentual seja subestimado, já que é um transtorno frequentemente secreto e nenhum estudo epidemiológico amplo para essa faixa etária foi realizado ${ }^{(14)}$.

O objetivo do presente trabalho foi descrever dois casos de tricotilomania acompanhados no serviço de Psiquiatria Infantil da Faculdade de Medicina de Botucatu da Universidade Estadual Paulista (Unesp), destacando suas características clínicas e discutindo as implicações do diagnóstico precoce para a evolução das pacientes.

\section{Descrição dos casos}

\section{Caso 1}

Paciente do sexo feminino, 11 anos, encaminhada pela psicóloga de sua escola, com queixa de arrancar os cabelos. Os pais contavam que, sem nenhum desencadeante ambiental, há cinco meses a garota havia começado a arrancar fios de cabelo, apresentando extensas áreas de alopecia em todo o couro cabeludo. A menina referia sentir uma necessidade urgente e incontrolável de arrancar os cabelos, seguida de alívio momentâneo. Consultou-se com dois pediatras, que orientaram a mãe sobre a benignidade do quadro e que, com o tempo, a menina espontaneamente interromperia esse comportamento. A família negava qualquer dificuldade de relacionamento em casa ou em outros contextos sociais. A garota se relacionava bem com seus amigos e, embora não deixasse de frequentar a escola, ficava mais isolada do que o habitual, pois se incomodava com as perguntas a respeito de seu cabelo. A mãe notou que ela arrancava mais cabelos quando ficava ansiosa por algum motivo. Ao final da primeira consulta, depois de receber orientação sobre o transtorno e os riscos associados, a paciente revelou que engolia os pedacinhos da "raiz" ou bulbo dos cabelos. Negava, porém, sintomas gastrintestinais. Foi encaminhada para psicoterapia, introduzindo-se clomipramina gradualmente até a dose de $1,5 \mathrm{mg} / \mathrm{kg} / \mathrm{dia}$. Após cinco meses de tratamento, a paciente não apresentava mais nenhuma área de alopecia, todavia 


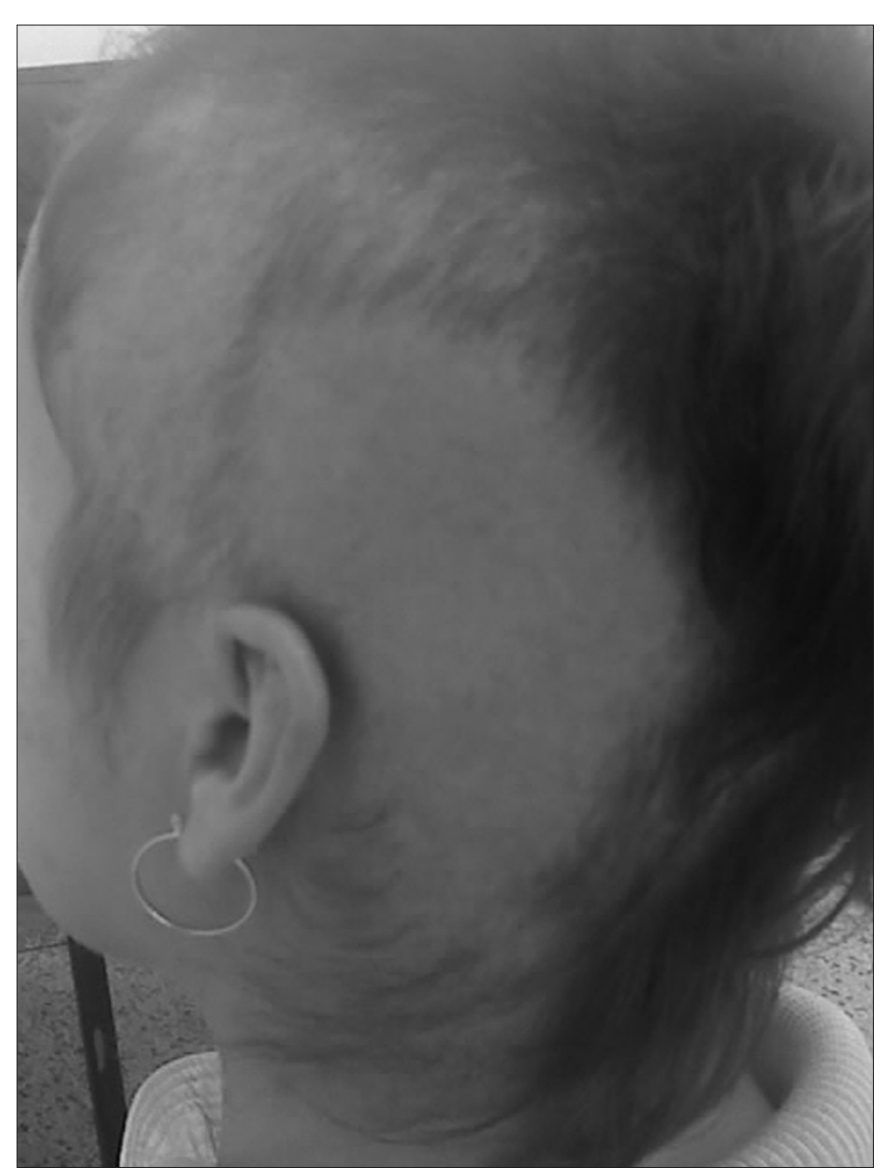

Figura 1 - Perda de cabelo em paciente com tricotilomania (Caso 2).

ainda experimentava esporadicamente alguma urgência em arrancar os cabelos. A pedido da mãe, foi encaminhada para acompanhamento homeopático para tratar de rinite, iniciando-se nessa época a regressão gradual da clomipramina. A menor foi reavaliada três, seis e 12 meses após a retirada completa da medicação alopática, apresentando excelente evolução. Em seu último retorno, um ano após a suspensão, não relatava novos episódios de tricotilomania, negando qualquer necessidade de arrancar os cabelos. Mantinha-se em acompanhamento homeopático.

\section{Caso 2}

Paciente do sexo feminino, com 12 anos de idade, apresentava desde os oito anos preocupações excessivas relacionadas ao medo de adquirir doenças e ao medo de contato com objetos vermelhos, pois "lembravam sangue" e poderiam estar contaminados. A mãe referia que a filha estava arrancando os cabelos há alguns meses, estando com extensas falhas no couro cabeludo (Figura 1). Não havia nenhum pensamento precedendo o ato de arrancar os cabelos, referindo apenas sentir uma necessidade incontrolável de fazê-lo repetidamente e um alívio passageiro após o ato. Os pensamentos obsessivos sobre doenças ocupavam muitas horas de seu dia e não havia nada que os aliviasse. Abandonara a escola pelo medo de adquirir doenças e pela vergonha das áreas de alopecia. Porém, demorou quase um ano para ser encaminhada a um serviço de saúde mental. Mãe e filha negavam ingestão dos fios de cabelo. Foi introduzida clomipramina, até a dose de $2,0 \mathrm{mg} / \mathrm{kg} / \mathrm{dia}$, com boa evolução do sintoma. Após seis meses não apresentava mais falhas no couro cabeludo, mas permanecia o medo de adquirir doenças e de voltar à escola. Foi acompanhada também em psicoterapia, conseguindo voltar a frequentar as aulas um ano após o início do tratamento. Manteve-se em uso da medicação, com controle total da tricotilomania e dos sintomas obsessivo-compulsivo após dois anos do início do quadro.

\section{Discussão}

A vergonha do comportamento e das áreas de alopecia, como descrita nesses casos, é bastante frequente na tricotilomania, o que faz com que os pacientes demorem para procurar ajuda, ocultando (ou negando) seus sintomas dos familiares, amigos e profissionais de saúde. Em estudo com pacientes tricotilomaníacos, observou-se que $40 \%$ deles nunca haviam sido diagnosticados e que $58 \%$ nunca haviam sido tratados ${ }^{(15)}$.

Não há consenso quanto à epidemiologia em adultos e crianças, já que inexistem estudos populacionais para tricotilomania. Em um estudo com 2.579 estudantes de universidades americanas, 0,6\% dos pesquisados tinham manifestado a doença em algum momento da vida, pelos critérios do Manual Diagnóstico e Estatístico de Transtornos Mentais (DSM-III-R) $)^{(16)}$.

Como o transtorno se inicia geralmente na infância e adolescência, o pediatra apresenta grande probabilidade de ser o primeiro profissional a ter contato com o paciente portador de tricotilomania. Nesse sentido, ganha importância a diferenciação com as formas benignas (leves e transitórias) do comportamento de arrancar cabelos, observadas em crianças menores ${ }^{(5)}$. O diagnóstico precoce pode minimizar as complicações sociais decorrentes da tricotilomania e mesmo impedir que o quadro evolua para tricobezoar, quando há tricofagia associada. Em torno de $40 \%$ dos pacientes engolem ou mastigam os fios arrancados, sendo que $40 \%$ destes podem apresentar sintomas clínicos secundários ao tricobezoar ${ }^{(17)}$.

Outro aspecto que merece ser ressaltado é que a tricotilomania pode não ser primária, mas se configurar como um 
sintoma de outro transtorno psiquiátrico e, quando isso ocorre, em geral aumenta a probabilidade de o paciente procurar ajuda especializada. Como dito acima, ha relato de tricotilomania associada a uma serie de diagnósticos psiquiátricos ${ }^{(10-}$ ${ }^{13)}$, podendo até mesmo ocorrer em paciente com transtorno afetivo unipolar, durante as fases de depressão ${ }^{(11)}$. Porém, a tricotilomania associada ao transtorno obsessivo-compulsivo (TOC) é uma das apresentações mais frequentes ${ }^{(18)}$. Em familiares de portadores de tricotilomania, a prevalência de TOC é mais elevada do que em famílias de controles normais, dando sustentação à hipótese de que esses transtornos podem pertencer a um mesmo espectro psicopatológico ${ }^{(19)}$. Um estudo neurofisiológico ${ }^{(20)}$ comparou pacientes com TOC e pacientes com tricotilomania, mostrando que, embora existam diferenças, há aspectos partilhados pelos dois grupos, como os problemas com memória espacial, por exemplo. Corrobora ainda o fato de vários casos de tricotilomania responderem a antidepressivos inibidores da recaptação da serotonina e à terapia cognitivo-comportamental, como ocorre no TOC ${ }^{(12)}$.

No entanto, nem sempre o tratamento da tricotilomania resulta em sucesso terapêutico. Para os pacientes que não respondem aos tratamentos acima descritos, várias abordagens alternativas são propostas, como o uso de neurolépticos e de estabilizadores do humor ${ }^{(1,12,21)}$. A raridade do transtorno e o fato de muitas vezes ser secreto dificultam a realização de ensaios clínicos controlados para melhor avaliação da resposta terapêutica, tanto farmacológica quanto psicoterápica ${ }^{(1)}$. Bloch et al realizaram uma revisão sistemática para comparar a eficácia da terapia comportamental e o uso de antidepressivos ${ }^{(22)}$. Apenas sete estudos preencheram os critérios de inclusão, sendo que a terapia comportamental foi superior ao uso da clomipramina e esta, superior ao placebo.

Na presença de sinais de alopecia, deve-se conduzir uma investigação ativa, questionando explicitamente, mas de modo acolhedor, o comportamento de arrancar cabelos e pelos

\section{Referências bibliográficas}

1. Tay YK, Levy ML, Metry DW. Trichotillomania in childhood: case series and review. Pediatrics 2004;113:e494-8.

2. Organização Mundial da Saúde, OMS. Classificação dos transtornos mentais e do comportamento da CID-10: descrições clínicas e diretrizes diagnósticas. 10a ed. Porto Alegre: Artmed; 1993.

3. Pereira JM. Compulsive trichoses. An Bras Dermatol 2004;79:609-18.

4. American Psychiatric Association. DSM-IV: Manual diagnóstico e estatístico de transtornos mentais. 4a ed. Porto Alegre: Artmed; 2002.
Quadro 1 - Perguntas que não podem faltar na anmnese.

1. Você tem o "hábito" de arrancar cabelos, cílios, sobrancelhas ou pelos de alguma parte do corpo?

2. Às vezes, você percebe que esse hábito é incontrolável? Descreva como acontece.

3. Por quanto tempo você fica arrancando os cabelos/ pelos?

4. Arrancar cabelos/pelos Ihe preocupa? Costuma, em função disso, deixar de fazer coisas ou sair de casa?

5. Você chega a se isolar em função desse comportamento?

6. Você se lembra de quando começou a arrancar cabelos/pelos, ou consegue associar tal costume com algum fato ou situação de sua vida? Descreva.

Fonte: Toledo e Cordás ${ }^{17}$, reproduzido com autorização.

do corpo. Toledo e Cordás ${ }^{(17)}$ propuseram seis perguntas que podem auxiliar o médico no diagnóstico, quando há suspeita de tricotilomania (Quadro 1). Enquanto nas formas benignas que ocorrem na primeira infância, mudanças da rotina de cuidados podem ser suficientes para abolir os sintomas, nas manifestações mais tardias é muitas vezes necessário o uso de medicamentos ou psicoterapia.

Embora as melhores formas de tratamento ainda não estejam bem estabelecidas, a importância do diagnóstico precoce da tricotilomania é consensual, tanto pelas potenciais complicações clínicas quanto pelo prejuízo psicossocial. Esse fato torna extremamente importante a compreensão não só da tricotilomania, mas também das inúmeras alterações psicopatológicas que podem ocorrer no continuum do desenvolvimento das crianças. Em relação à tricotilomania, é fundamental que os pediatras estejam atentos para diferenciar o quadro benigno e transitório de arrancar cabelos que ocorre na primeira infância e a tricotilomania de início mais tardio, muitas vezes de evolução crônica, potencialmente grave e geradora de sofrimento e impacto relevantes.

5. Santhanam R, Fairley M, Rogers M. Is it trichotillomania? Hair pulling in childhood: a developmental perspective. Clin Child Psychol Psychiatry 2008;13:409-18.

6. Lewin AB, Piacentini J, Flessner CA, Woods DW, Franklin ME, Keuthen NJ et al. Depression, anxiety, and functional impairment in children with trichotillomania. Depress Anxiety 2009:26;521-7.

7. Grant JE, Odlaug BL. Clinical characteristics of trichotillomania with trichophagia. Compr Psychiatry 2008;49:579-84. 
8. Godoy $A Q$, Pontes RM, Godoy AR. Úlcera gástrica terebrante e pancreatite aguda provocadas por tricobezoar. Rev Col Bras Cir 2004;31:71-2.

9. Aguilar-Arauz M, Rodriguez-Gutierrez F, Hernandez-Mena C. Síndrome de Rapunzel: reporte de un caso. Acta Med Costarric 2003;45:80-3.

10. Sah DE, Koo J, Price VH. Trichotillomania. Dermatol Ther 2008;21: 13-21.

11. Kerr-Corrêa F, Torres AR, Bocuto C, Cavicchioli MG. Tricotilomania e outras doenças psiquiátricas. Rev ABP-APAL 1992;14:111-4.

12. Chamberlain SR, Menzies L, Sahakian BJ, Fineberg NA. Lifting the veil on trichotillomania. Am J Psychiatry 2007;164:568-74.

13. Odlaug BL, Grant JE. Trichotillomania and Pathologic Skin Picking: clinical comparison with an examination of comorbidity. Ann Clin Psychiatry 2008;20:57-63.

14. Keuthen NJ, O'Sullivan RL, Goodchild P, Rodriguez D, Jenike MA, Baer L. Retrospective review treatment outcome for 63 patients with trichotillomania. Am J Psychiatry 1998;155:560-1.

15. Cohen LJ, Stein DJ, Simeon D, Spadaccini E, Rosen J, Aronowitz B et al. Clinical profile, comorbidity, and treatment history in 123 hair pullers: a survey study. J Clin Psychiatry 1995;56:319-26.
16. Christenson GA, Pyle RL, Mitchell JE. Estimated lifetime prevalence of trichotillomania in college students. J Clin Psychiatry 1991;52:415-7.

17. Toledo EL, Cordás TA. Tricotilomania. In: Abreu C, Tavares H, Cordás TA, editors. Manual clínico dos transtornos do controle dos impulsos. Porto Alegre: Artmed; 2007. p. 63-77.

18. Mercadante MT, Rosário-Campos MC, Quarantini LC, Sato FP. The neurobiological bases of obsessive-compulsive disorder and Tourette syndrome. J Pediatr (Rio J) 2004;80:S35-44.

19. Lochner C, Seedat S, Du Toit PL, Nel DG, Niehaus DJ, Sandler R et al. Obsessive-compulsive disorder and trichotillomania: a phenomenological comparison. BMC Psychiatry 2005;5:2.

20. Chamberlain SR, Fineberg NA, Blackwell AD, Clark L, Robbins TW, Sahakian BJ. A neuropsychological comparison of obsessive-compulsive disorder and trichotillomania. Neuropsychologia 2007;45:654-62.

21. Adewuya EC, Zinser W, Thomas C. Trichotillomania: a case of response to valproic acid. J Child Adolesc Psychopharmacol 2008;18:533-6.

22. Bloch MH, Landeros-Weisenberger A, Dombrowski P, Kelmendi B, Wegner R, Nudel $\mathrm{J}$ et al. Systematic review: pharmacological and behavioral treatment for trichotillomania. Biol Psychiatry 2007;62:839-46. 